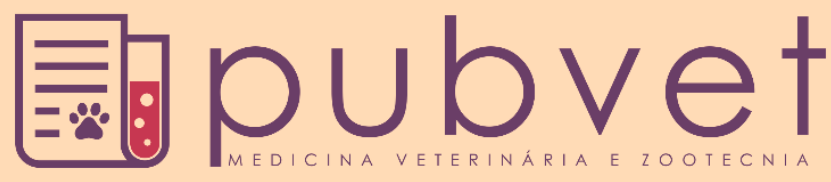

https://doi.org/10.31533/pubvet.v15n12a986.1-4

\title{
Fibropapilomatose em tartarugas marinhas: Revisão
}

\author{
Brenda Madruga Rosa ${ }^{1} \bullet$, Eduardo Gonçalves da Silva ${ }^{1 *} \bullet$, Michaela Marques Rocha ${ }^{1} \bullet$, \\ Caroline Castagnara Alves $^{1 \oplus}$, Francesca Lopes Zibetti ${ }^{1}{ }^{\ominus}$, Daniele Weber Fernandes ${ }^{1}{ }^{\ominus}$, Maysa \\ Seibert de Leão ${ }^{10}$, Thaís Cristina Vann ${ }^{1}{ }^{\circ}$, Amanda Leal de Vasconcellos $^{20}$, Paula Priscila \\ Correia $\operatorname{Costa}^{30}$ \\ ${ }^{I}$ Graduando de Medicina Veterinária, Universidade Federal de Pelotas, Pelotas-RS, Brasil \\ ${ }^{2}$ Professor da Universidade Estadual do Ceará, Departamento de Clínicas Veterinárias, Fortaleza-CE, Brasil \\ ${ }^{3}$ Professor da Universidade Federal de Pelotas, Departamento de Clínicas Veterinárias, Pelotas-RS, Brasil \\ *Autor para correspondência, E-mail: goncalves-eduardo@outlook.com
}

Resumo. A presente revisão tem por objetivo copilar informações já relatadas sobre a fibropapilomatose em tartarugas marinhas, doença altamente debilitante e potencialmente fatal, caracterizada pela formação de fibropapilomas cutâneos e fibromas viscerais, possuindo distribuição mundial, afetando principalmente tartarugas verdes. A etiologia dessa enfermidade ainda não foi estabelecida, mas a suspeita é de uma infecção viral que pode ocorrer associada a fatores ambientais. O número e tamanhos dos tumores variam entre cada indivíduo e a sua presença, em alguns casos dificulta a qualidade de vida desse animal. A confirmação ocorre por meio de diagnóstico histológico. A principal terapia empregada é a remoção cirúrgica dos papilomas.

Palavras chave: Excisão cirúrgica, histopatologia, quelônios, tumores

\section{Fibropapillomatosis in sea turtles: Review}

\begin{abstract}
The current work aims to present a review of the information already reported on fibropapillomatosis in sea turtles, a highly debilitating and potentially fatal disease, characterized by the formation of cutaneous fibropapilomas and visceral fibroids, having a worldwide distribution, mainly affecting green turtles. The etiology has not yet been established, but a viral infection that happens associated with environmental factors is suspected. The number and sizes of tumors vary between each individual and their presence, in some cases hindering the quality of life of this animal. Confirmation occurs through histological diagnosis. The main therapy used is the surgical removal of papillomas.
\end{abstract}

Keywords: Surgical excision, histopathology, chelonians, tumors

\section{Introdução}

No presente momento, existem sete espécies de tartarugas marinhas descritas e distribuídas ao longo dos oceanos, sendo que dessas apenas cinco ocorrem no litoral brasileiro: tartaruga-verde (Chelonia mydas), tartaruga-cabeçuda (Caretta caretta), tartaruga-de-couro (Dermochelys coriacea), tartaruga-depente (Eretmochelys imbricata) e tartaruga-oliva (Lepidochelis olivacea) (Amorim, 2010). Inúmeros fatores, como a destruição do habitat natural, foto poluição, pesca acidental, tráfego de veículos e predação de ovos, colocam em risco a conservação das tartarugas marinhas (Silva Júnior, 2016), somado a isso, doenças infecciosas, em especial a fibropapilomatose, também desempenham um papel importante na redução da população dessas espécies (Herbst, 1994).

O primeiro caso relatado foi descrito por Lucke em 1938 e Smith e Coates também em 1938 e ocorreu em uma tartaruga verde (Chelonia mydas) no estado da Flórida (EUA) (Matushima et al., 2001; Work 
\& Balazs, 1999), concomitante ao fato dessa espécie ser a mais afetada, a doença foi inicialmente denominada como Green turtle fibropapillomatosis (GTFP) (Amorim, 2010; Cubas et al., 2014). No Brasil, o primeiro caso registrado foi no ano de 1986, no estado do Espírito Santo, e desde o ano 2000 o Projeto Tamar ICMBio (Projeto Tartaruga Marinha - Instituto Chico Mendes de Conservação da Biodiversidade) tem relatado a presença da doença em suas áreas de monitoramento (Amorim, 2010; Cubas et al., 2014). A fibropapilomatose é uma enfermidade debilitante e é caracterizada por inúmeros tumores cutâneos benignos, divididos em três lesões proliferativas: papilomas cutâneos, fibromas e fibropapilomas (Herbst, 1994). Apesar do caráter benigno, a presença dos papilomas compromete a natação, visão, alimentação e/ou respiração das tartarugas marinhas (Amorim, 2010; Cubas et al., 2014; Divers \& Mader, 2005; Mader, 1996). O presente trabalho tem como objetivo realizar uma revisão de literatura acerca das informações já publicadas sobre fibropapilomatose em tartarugas marinhas.

\section{Epidemiologia}

A enfermidade afeta indivíduos juvenis, sub-adultos e adultos (Amorim, 2010), foi relatada com distribuição circuntropical mundial e em todos os oceanos (Aguirre, 1998). A principal espécie de tartaruga marinha acometida é a Chelonia mydas (tartaruga-verde), entretanto também foi relatada nas demais espécies existentes, como a Caretta caretta (tartaruga-cabeçuda), Lepidochelys olivacea (tartaruga-oliva), Dermochelys coriacea (tartaruga-de-couro), Eretmochelys imbricata (tartaruga-depente), e Lepidochelys kempii (tartaruga-de-kemp); na espécie Natator depressus (tartaruga-flatback) foram descritas lesões semelhantes ao fibropapiloma que não foram confirmadas histopalogicamente (Aguirre et al., 1999; Harshbarger, 1991; Herbst, 1994; Huerta et al., 2002; Silva Júnior, 2016).

A prevalência da doença varia entre 1,4\% a 90\% (Aguirre, 1998) e tem maior severidade em animais com peso entre 10 e $20 \mathrm{~kg}$ e é menos severa e frequente naqueles acima de $30 \mathrm{~kg}$ (Ehrhart, 1991). Há indícios de uma associação da alta prevalência com habitats marinhos costeiros e com a atividade antrópica próximo a áreas de captação de água (Amorim, 2010; Herbst, 1994).

\section{Etiologia}

O agente etiológico causador da fibropapilomatose ainda não está totalmente elucidado (Herbst et al., 1995). Os estudos indicam que a enfermidade está associada a uma infecção por um herpesvírus, além disso, a ocorrência também ocorre com ligação a vários fatores, como contaminantes químicos, radiação ultravioleta, presença de parasitos e imunossupressão (Cubas et al., 2014; Jones et al., 2016).

\section{Manifestação clínica e lesões}

A fibropapilomatose é uma enfermidade potencialmente fatal e de caráter debilitante para as tartarugas marinhas (Cubas et al., 2014; Jones et al., 2016). O número e tamanho dos papilomas variam entre cada indivíduo e seu tamanho pode atingir aproximadamente $30 \mathrm{~cm}$ de diâmetro (Herbst, 1994; Matushima et al., 2001). As lesões são descritas com aparência verrucosa e de consistência macia com coloração rosada, ou aspecto de couve flor e consistência dura com coloração cinza-esverdeado, sendo que essas podem apresentar ulceração (Amorim, 2010; Knöbl et al., 2011) e são comumente encontradas em todo o tecido tegumentar mole, como as nadadeiras, pescoço e região periocular (Divers \& Mader, 2005; Jacobson et al., 1989).

A presença dos papilomas na região ocular pode reduzir ou eliminar a visão do animal (Divers \& Mader, 2005; Jacobson et al., 1989), ademais a presença dos mesmos dificulta a locomoção, apreensão de alimentos e a respiração (Cubas et al., 2014; Divers \& Mader, 2005; Jones et al., 2016), ameaçando a sobrevivência do animal no seu habitat (Cubas et al., 2014; Jones et al., 2016) e aumentando a suscetibilidade ao encalhe (Aguirre et al., 1999). Os tumores viscerais surgem tardiamente em relação ao curso clínico da enfermidade (Herbst et al., 1999) e são comumente encontrados nos pulmões, rins, coração, trato gastrointestinal e fígado (Divers \& Mader, 2005; Herbst, 1994).

As tartarugas portadoras da enfermidade apresentam hipoproteinemia e anemia (Aguirre et al., 1999; Cubas et al., 2014), associado à linfocitopenia e neutrofilia indicando uma supressão do sistema imunológico. Ademais, os valores bioquímicos apontam que esses animais sofriam de uma enfermidade crônica e debilitante (Aguirre et al., 1995). Os animais com estado clínico avançado podem apresentar 
condições avançadas de acidose e um desequilíbrio na proporção de cálcio e fósforo (Aguirre, 1998), além um declínio nos níveos de colesterol e triglicerídeos, que podem estar relacionados com à perda de escore corporal e fome (Aguirre \& Balazs, 2000).

\section{Diagnóstico}

O diagnóstico é realizado clinicamente pela visualização dos papilomas (Divers \& Mader, 2005). Para tumores viscerais o diagnóstico ocorre por meio de radiografias, ressonância magnética, tomografia computadorizada, laparoscopia ou necropsia (Divers \& Mader, 2005; Page-Karjian et al., 2014) e apresentam prognóstico reservado (Knöbl et al., 2011). A confirmação do diagnóstico ocorre por meio do exame histológico (Knöbl et al., 2011) e é caracterizada por degeneração e necrose dos queratinócitos, inclusão citoplasmáticas eosinofílicas, proliferação estromal hiperplásica e proliferação epidermal (Matushima et al., 2001).

\section{Tratamento}

Não existe uma terapia especifica para tratar a fibropapilomatose (Divers \& Mader, 2005). O principal procedimento terapêutico empregado é a excisão cirúrgica dos papilomas (Cubas et al., 2014; Futema, 2002; Mader, 1996). Entretanto, é importante ressaltar que para animais debilitados a anestesia geral e o procedimento invasivo são fatores de risco (Dastre, 2017). No caso de tartarugas com a enfermidade leve, espera-se a regressão espontânea dos papilomas (Divers \& Mader, 2005; Mader, 1996). Além da excisão cirúrgica, outra opção empregada como tratamento seria a criocirurgia, e a mesma pode causar a regressão dos tumores e estimulação do sistema imune (Dastre, 2017).

\section{Considerações finais}

A fibropapilomatose é uma enfermidade de grande importância para a conservação das tartarugas marinhas, visto que apresenta grande prevalência, por isso é fundamental maiores estudos a fim de avaliar maiores opções de tratamento e acerca do real agente etiológico.

\section{Referências bibliográficas}

Aguirre, A A. (1998). Fibropapilomas en Tortugas Marinas: un Taller del XVIII Simpósio anual sobre la biología y la conservación de las tortugas marinas. Noticiero de Tortugas Marinas., 82, 10-12.

Aguirre, A Alonso, Balazs, G. H., Spraker, T. R., \& Gross, T. S. (1995). Adrenal and hematological responses to stress in juvenile green turtles (Chelonia mydas) with and without fibropapillomas. Physiological Zoology, 68(5), 831-854. https://doi.org/10.2307/30163934.

Aguirre, A Alonso, Spraker, T. R., Chaves, A., Toit, L., Eure, W., \& Balazs, G. H. (1999). Pathology of fibropapillomatosis in olive ridley turtles Lepidochelys olivacea nesting in Costa Rica. Journal of Aquatic Animal Health, 11(3), 283-289. https://doi.org/10.1577/15488667(1999)011<0283:POFIOR>2.0.CO;2.

Aguirre, A. Al, \& Balazs, G. H. (2000). Blood biochemistry values of green turtles, Chelonia mydas, with and without fibropapillomatosis. Comparative Haematology International, 10(3), 132-137. https://doi.org/10.1007/s005800070004.

Amorim, D. B. (2010). Fibropapilomatose em tartarugas marinhas. Universidade Federal do Rio Grande do Sul.

Cubas, Z. S., Silva, J. C. R., \& Dias, J. L. C. (2014). Tratado de animais selvagens-medicina veterinária. Editora Roca.

Dastre, M. (2017). Métodos convencionais para o tratamento de fibropapilomatose em testudines marinhos: revisão sistemática. Universidade Estadual Paulista (UNESP).

Divers, S. J., \& Mader, D. R. (2005). Reptile medicine and surgery. Elsevier Health Sciences.

Ehrhart, L. M. (1991). Fibropapillomas in green turtles of the Indian River Lagoon, Florida: distribution over time and area. National Oceanic and Atmospheric Administration, 156, 59-61.

Futema, F. (2002). Avaliação pré-anestésica. Roca.

Harshbarger, J. C. (1991). Sea turtle fibropapilloma cases in the registry of tumors in lower animals. 
Research Plan for Marine Turtle Fibropapilloma, 156, 63-70.

Herbst, L. H. (1994). Fibropapillomatosis of marine turtles. Annual Review of Fish Diseases, 4, 389425. https://doi.org/10.1016/0959-8030(94)90037-X.

Herbst, L. H., Jacobson, E. R., Klein, P. A., Balazs, G. H., Moretti, R., Brown, T., \& Sundberg, J. P. (1999). Comparative pathology and pathogenesis of spontaneous and experimentally induced fibropapillomas of green turtles (Chelonia mydas). Veterinary Pathology, 36(6), 551-564. https://doi.org/10.1354/vp.36-6-5519.

Herbst, L. H., Jacobson, E. R., Moretti, R., Brown, T., Sundberg, J. P., \& Klein, P. A. (1995). Experimental transmission of green turtle fibropapillomatosis using cell-free tumor extracts. Diseases of Aquatic Organisms, 22(1), 1-12. https://doi.org/10.3354/dao022001.

Huerta, P., Pineda, H., Aguirre, A., Spraker, T., Sarti, L., \& Barragan, A. (2002). First confirmed case of fibropapilloma in a leatherback turtle (Dermochelys coriacea). Proceedings of the 20th Annual Symposium on Sea Turtle Biology and Conservation., 193.

Jacobson, E. R., Mansell, J. L., Sundberg, J. P., Hajjar, L., Reichmann, M. E., Ehrhart, L. M., Walsh, M., \& Murru, F. (1989). Cutaneous fibropapillomas of green turtles (Chelonia mydas). Journal of Comparative Pathology, 101(1), 39-52. https://doi.org/10.1016/0021-9975(89)90075-3.

Jones, K., Ariel, E., Burgess, G., \& Read, M. (2016). A review of fibropapillomatosis in green turtles (Chelonia mydas). The Veterinary Journal, 212, 48-57. https://doi.org/10.1016/j.tvj1.2015.10.041.

Knöbl, T., Reiche, R., \& Menão, M. C. (2011). Fibropapilomatose em tartarugas marinhas. Neotropical Biology \& Conservation, 6(1), 64-69. https://doi.org/10.4013/nbc.2011.61.08.

Mader, D. R. (1996). Reptile Medicine and Surgery-E-Book. Elsevier Health Sciences.

Matushima, E. R., Longatto Filho, A., Loretto, C., Kanamura, C. T., Sinhorini, I. L., Gallo, B., \& Baptistolle, C. (2001). Cutaneous papillomas of green turtles: a morphological, ultra-structural and immunohistochemical study in Brazilian specimens. Brazilian Journal of Veterinary Research and Animal Science, 38, 51-54. https://doi.org/10.1590/S1413-95962001000200001.

Page-Karjian, A., Norton, T. M., Krimer, P., Groner, M., Nelson, S. E., \& Gottdenker, N. L. (2014). Factors influencing survivorship of rehabilitating green sea turtles (Chelonia mydas) with fibropapillomatosis. Journal of Zoo and Wildlife Medicine, 45(3), 507-519. https://doi.org/10.1638/2013-0132R1.1.

Silva Júnior, E. S. (2016). Incidência de fibropapilomatose em tartarugas marinhas na Bacia Potiguar $R N / C E$. Universidade Federal do Rio Grande do Norte.

Work, T. M., \& Balazs, G. H. (1999). Relating tumor score to hematology in green turtles with fibropapillomatosis in Hawaii. Journal of Wildlife Diseases, 35(4), 804-807. https://doi.org/10.7589/0090-3558-35.4.804.

Histórico do artigo:

Recebido: 6 de agosto de 2021

Aprovado: 1 de setembro de 2021

Disponível on-line: 12 de novembro de 2021
Licenciamento: Este artigo é publicado na modalidade Acesso Aberto sob a licença Creative Commons Atribuição 4.0 (CC-BY 4.0), a qual permite uso irrestrito, distribuição, reprodução em qualquer meio, desde que o autor e a fonte sejam devidamente creditados. 